\title{
An APD for the efficient detection of the fast scintillation component of $\mathrm{BaF}_{2}$
}

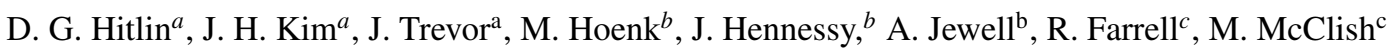 \\ ${ }^{a}$ Lauritsen Laboratory, Caltech, Pasadena CA, USA \\ ${ }^{b}$ Jet Propulsion Laboratory, Caltech, Pasadena CA, USA \\ ${ }^{c}$ RMD Inc. Watertown MA, USA
}

\begin{abstract}
Barium fluoride crystals are the baseline choice for the calorimeter of the Mu2e experiment at Fermilab. By the fast (decay time $0.9 \mathrm{~ns}$ ) $220 \mathrm{~nm}$ scintillation component and discriminating against the larger slow (decay time 630ns) $300 \mathrm{~nm}$ component, it is possible to build a radiation-hard calorimeter with good energy and time resolution and high rate capability. This requires a solid state photosensor with high quantum efficiency at $220 \mathrm{~nm}$, discrimination against the $300 \mathrm{~nm}$ component and good rise and decay times. Progress on the development of such a sensor is presented.
\end{abstract}

Keywords: barium fluoride, photosensors, APD, solar blind, superlattice, atomic layer deposition PACS: 29.40.Cs, 29.40.Gx

\section{Introduction}

The Mu2e experiment at Fermilab[1] employs a crystal ${ }^{24}$ calorimeter for the identification of $105 \mathrm{MeV}$ electrons pro- ${ }^{25}$ duced in the rare process of muon-to-electron conversion on ${ }^{26}$ an aluminium nucleus. The calorimeter must operate in a high ${ }^{27}$ rate, high radiation, environment, in the presence of substantial background from muon decay and muon capture. Several crystals have been considered for the calorimeter. The initial concept employed $\mathrm{PbWO}_{4}$. Problems of low light output and the variation of light output with instantaneous rate motivated ${ }_{33}^{32}$ the design of a calorimeter based on LYSO[2], which is an excellent match to the problem, having high light output, fast scintillation decay time and good radiation hardness. However, the price of LYSO has increased substantially[3] in the last few years, making it too expensive for the Mu2e application. An interesting alternative is barium fluoride, $\mathrm{BaF}_{2}$, which has two scintillation components, one at $220 \mathrm{~nm}$ has a decay time of 0.9 $\mathrm{ns}$, while the larger component at $300 \mathrm{~nm}$ has a decay time of $630 \mathrm{~ns}[4]$. Figure 1 shows the $\mathrm{BaF}_{2}$ scintillation time distribution.

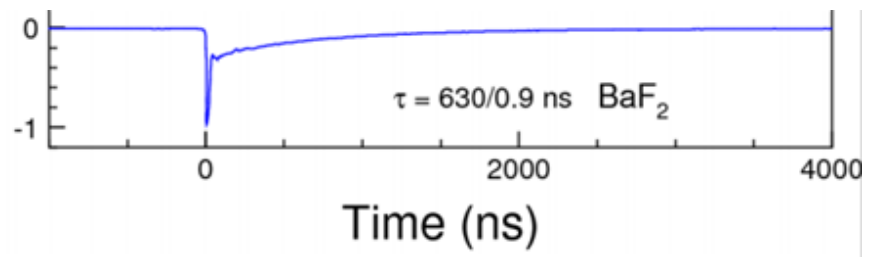

Figure 1: The fast and slow scintillation components of $\mathrm{BaF}_{2}$

The existence of the very fast scintillation component in $\mathrm{BaF}_{2}$ makes this crystal an attractive candidate for high rate ap-

Email address: hitlin@caltech.edu (D. G. Hitlin ${ }^{a}$, J. H. Kim ${ }^{a}$, J. Trevor)

Preprint submitted to Elsevier plications such as the Mu2e calorimeter, in both its initial and possible upgrade phases. The $\mathrm{BaF}_{2}$ scintillation spectrum is shown in Figure 2. $\mathrm{BaF}_{2}$ was thoroughly investigated as a candidate for the calorimeter of the GEM detector at the SSC[5] more than two decades ago, and has, in fact, been employed in the TAPS experiment[6] at Mainz, which does not have a magnetic field. For Mu2e to make full use of the high rate capability afforded by the fast decay time component at $220 \mathrm{~nm}$, however, requires a means of rejecting the much more intense slow component at $300 \mathrm{~nm}$ with a readout device that can operate in a 1T magnetic field. Solar blind photomultipiers have adequate quantum efficiency at $220 \mathrm{~nm}$, and some rejection at $300 \mathrm{~nm}$, but are not suitable for use in a magnetic field.

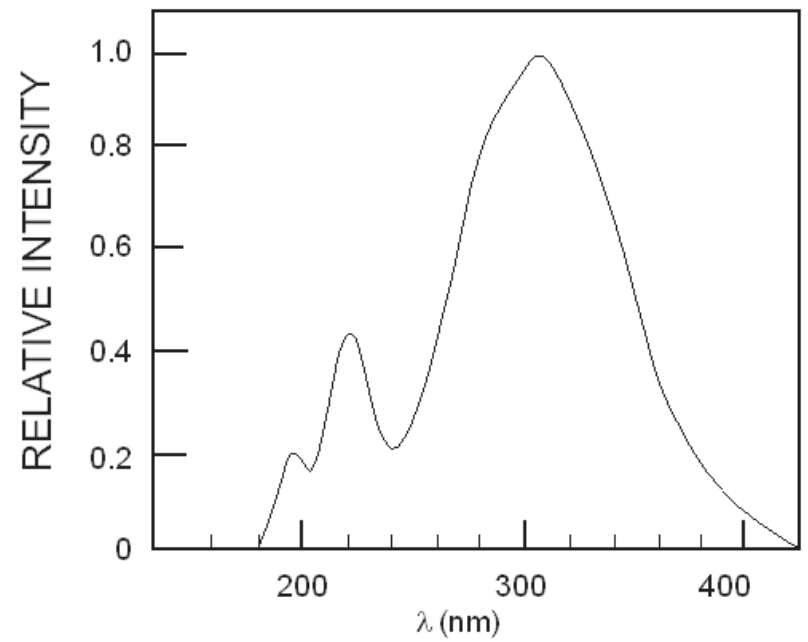

Figure 2: The $\mathrm{BaF}_{2}$ fast scintillation component at $220 \mathrm{~nm}$ comprises $\sim 15 \%$ of the light, the slow component at $300 \mathrm{~nm}, \sim 85 \%$

There are approaches to making use of the fast $220 \mathrm{~nm}$ component of $\mathrm{BaF}_{2}$ : reducing the size of the $300 \mathrm{~nm}$ component 
through doping and/or finding a suitable readout device that is insensitive to the slow component while retaining adequate quantum efficiency for the fast component. It is possible to reduce the $300 \mathrm{~nm}$ emission component without too much effect on the $220 \mathrm{~nm}$ component by doping with rare earths such as lanthanum[7], but this is not a large enough reduction to solve the problem at hand. We have begun a study of this type of doping, mainly aimed at understanding the radiation hardness of dope $\mathrm{BaF}_{2}$ crystals.

Our main focus has been on developing UV sensitive solid state photosensors. There are commercial devices with extended UV response, but no large area devices that can discriminate between the wavelengths of the fast and slow components of $\mathrm{BaF}_{2}$ emission. We have thus formed a Caltech/JPL/RMD collaboration to develop such a device.

\section{Superlattice deposition}

Typical large area APDs have poor quantum efficiency in the $\mathrm{BaF}_{2}$ spectral region. However, APDs and SiPMs/MPPCs from Hamamatsu and RMD made without the normal protective epoxy coating, and therefore somewhat fragile, have quantum efficiencies in the $200 \mathrm{~nm}$ region of $17 \%$ [8]. These devices cannot, however, discriminate between the $220 \mathrm{~nm}$ fast component and $300 \mathrm{~nm}$ slow components of $\mathrm{BaF}_{2}$. The presence of the slow component limits the rate capability of the calorimeter, and can therefore be an issue in high background conditions.

Our approach is to transform a large-area $\left(9 \times 9 \mathrm{~mm}^{2}\right) \mathrm{RMD}$ APD [9], which has high gain (up to 2000) and low capacitance $0.7 \mathrm{pf} / \mathrm{mm}^{2}$, into a superlattice-doped APD [10] and to incorporate an atomic layer deposition (ALD) antireflection filter [11] that provides $60 \%$ quantum efficiency at $220 \mathrm{~nm}$ and $0.1 \%$ efficiency at $300 \mathrm{~nm}$, thereby enabling us to obtain a larger number of photoelectrons/ $\mathrm{MeV}$, and also to take full advantage of the fast decay time component of $\mathrm{BaF}_{2}$.

Superlattice (2D) doping, a JPL-developed surface passivation technique, produces stable surfaces on silicon photosensors. These techniques were developed to overcome surface damage due to ultraviolet radiation in satellite instrumentation. The subsurface structures are formed using a combination of molecular beam epitaxy and controlled crystalline silicon growth. After growing a thin layer of undoped silicon, a monolayer of boron is deposited, then another silicon layer is ${ }^{93}$ grown, and the process is repeated up to four times. The result- ${ }^{94}$ ing subsurface structure, with high quality self-organized layers ${ }^{95}$ of boron atoms at densities of $\sim 2 \times 10^{14} / \mathrm{cm}^{2}$ (see Figure 3),

The reason for the low QE achieved by most solid state de- 96 vices in the UV region is two-fold: in typical devices there is an undepleted region of tens of microns between the $\mathrm{Si} / \mathrm{SiO}_{2}{ }_{97}$ passivation region on the absorbing surface and the depletion 98 region that is in close proximity to the avalanche region. Due 99 to quantum exclusion, the superlattice structure suppresses re-100 combination of charge at the surface, thereby improving the $\mathrm{QE}_{101}$ in the $220 \mathrm{~nm}$ region to close to the theoretical maximum. The 102 greatly reduced undepleted region of the superlattice-doped de-103 vice also produces substantially improved timing characteris-104 tics (see Figure 4). Second, most devices have a protective resin ${ }_{105}$

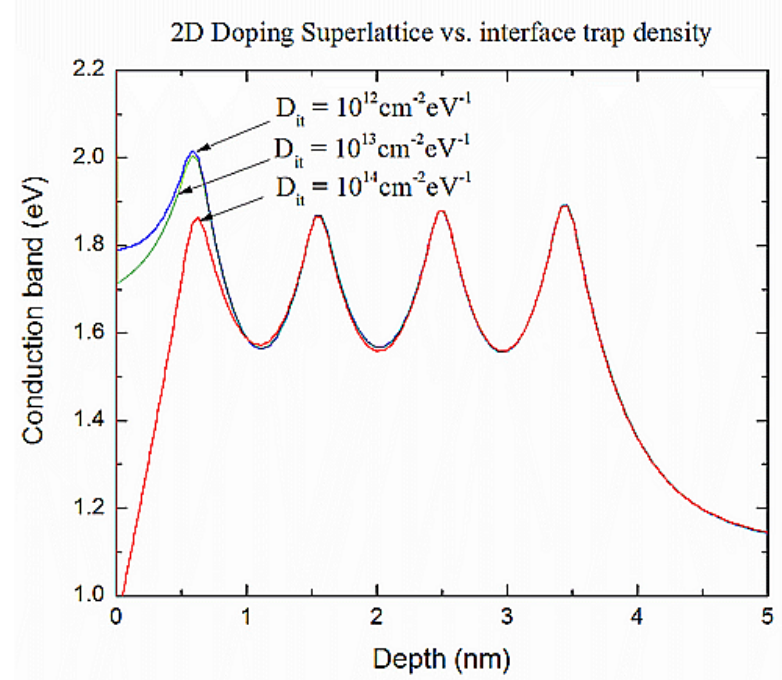

Figure 3: A four-layer superlattice grown by MBE, in which four 2D-doped layers are separated by $1 \mathrm{~nm}$. This $2 \mathrm{D}$ doping creates a maximum field of $10^{7}$ $\mathrm{V} / \mathrm{cm}$. The $2 \mathrm{D}$ doping superlattice forms a surface depletion layer with a fixed width of less than $1 \mathrm{~nm}$, which is stable against interface trap densities in excess of $10^{14} \mathrm{~cm}^{-3}$, enabling the detector to remain stable even when the surface is severely damaged by irradiation by DUV radiation.

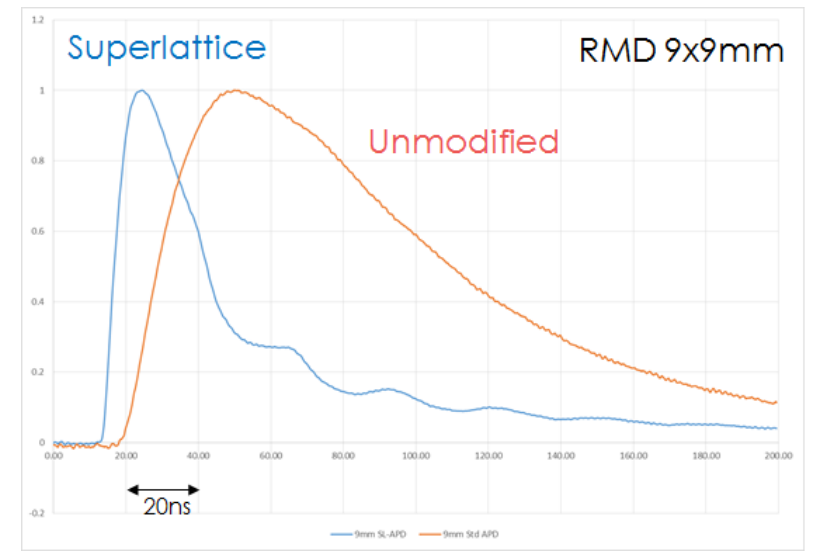

Figure 4: Response of unmodified and superlattice-doped $9 \times 9 \mathrm{~mm}^{2} \mathrm{RMD}$ APDs.

layer that lowers the net quantum efficiency. This layer is not required in our device, which is passivated and protected by the creation of an atomic layer deposition filter described below.

\section{Atomic layer deposition filter}

It then remains to apply a multilayered atomic layer deposition (ALD) coating to serve as a bandwidth-reducing interference and antireflection filter. These films, in our case alternating layers of aluminum and aluminum oxide, are created by forming a series of single atomic layers through selflimiting chemical reactions with the substrate. This process produces uniform pinhole-free layers of precise thickness. Figure 5 shows the calculated transmission of any early version of such a filter as a function of wavelength. For a five-layer ALD 
coating, the $\mathrm{QE}$ at normal incidence for the fast component of ${ }_{118}$ $\mathrm{BaF}_{2}$ is close to $70 \%$, and the extinction at the slow component ${ }_{119}$ wavelength is nearly complete. The transmission of the filter is ${ }_{120}$ a function of the incident angle; this response is shown in Fig-121 ure 3 for the current version of the five-layer interference filter, 122 which has response extended to below $200 \mathrm{~nm}$.

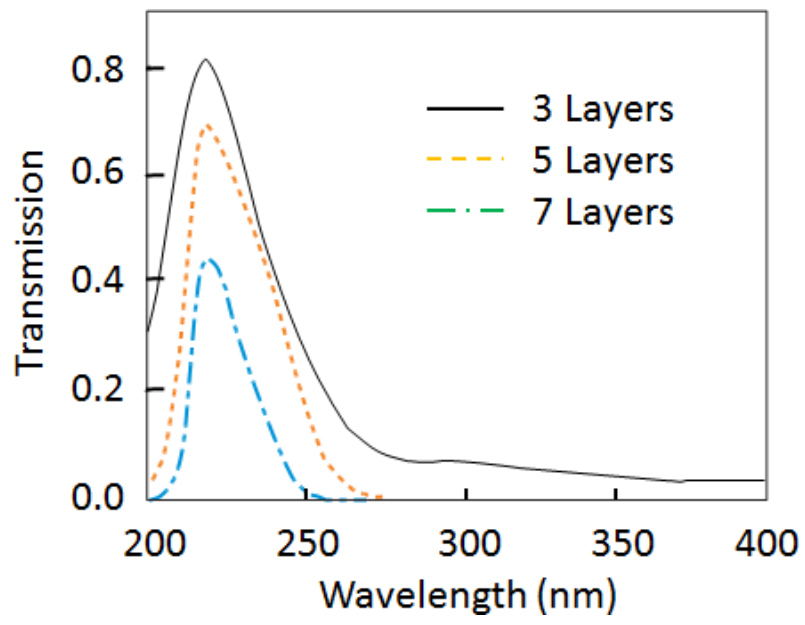

Figure 5: Quantum efficiency (QE) as a function of wavelength for the original three, five seven layer ALD filter design.

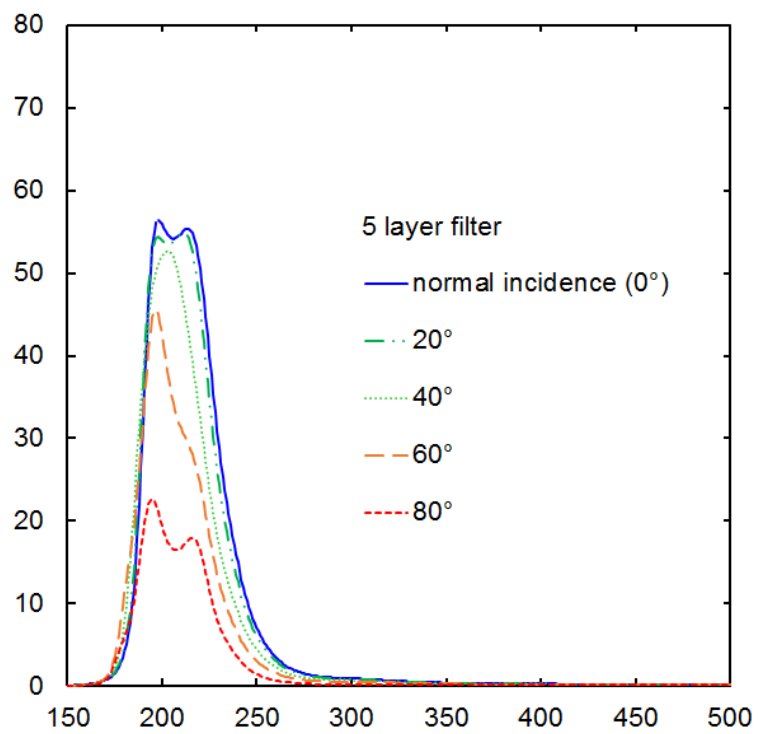

Figure 6: Response of an improved five layer ALD filter as a function of angle of incidence and wavelength.

\section{Electrical performance}

We have produced several versions of superlattice/ALD- ${ }_{-148}^{147}$ modified RMD APDs thus far. The anticipated filter response ${ }^{149}$ and quantum efficiency improvements have been experimen- ${ }^{150}$ tally demonstrated. The devices, however, have somewhat $\mathrm{el}^{151}$ evated dark current and noise, as seen in Figure 6. Operation ${ }_{153}$ at somewhat reduced temperatures improves the noise performance substantially: at $\sim 25^{\circ}$ below ambient, the noise of the superlattice/ALD-modified devices is equivalent to that of standard devices at room temperature.

Several process variations are being investigated to reduce the dark current and associated noise. These include variation of the parameters of the superlattice deposition layers, modification of the first dielectric layer of the ALD filter and changes to the electrical contact geometry. The current devices are nonetheless suitable for deployment in the Mu2e calorimeter.

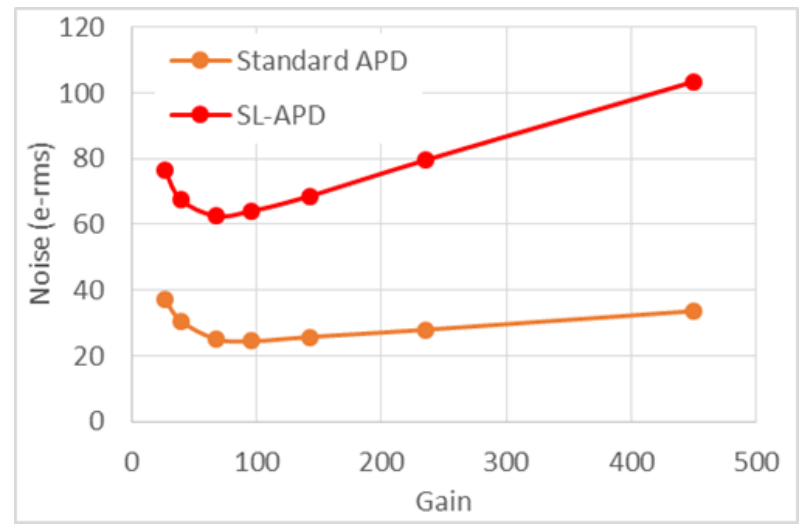

Figure 7: Noise in rms electrons of a standard and a superlattice-doped RMD APD as a function of gain.

\section{Conclusion}

We have described the development of a solar-blind avalanche photodiode suitable for the readout of the fast scintillation component of barium fluoride. The device, a modified RMD APD with a superlattice-doped region and an integrated atomic layer deposition filter, has high quantum efficiency for the $220 \mathrm{~nm}$ fast component, strong extinction for the $300 \mathrm{~nm}$ slow component and excellent timing performance.

\section{Acknowledgments}

The development of this UV sensitive, solar-blind APD a collaborative effort of Caltech, Jet Propulsion Laboratory and RMD Inc., under SBIR grant DE-SC0011316 and with NASA and DOE funds.

\section{References}

[1] L. Bartoszek et al. [Mu2e Collaboration], arXiv:1501.05241 [physics.ins-det].

[2] G. Pezzullo, et al., JINST 9, C03018 (2014).

[3] M. Dalton and W. Mauldin, The Wall Street Jounal, March 26, 2014.

[4] M. Laval, M. Moszynski, R. Allemand, E. Cormoreche, P . Guinet, R. Odru and J. Vacher, Nucl. Instrum. Meth. 206, 169 (1983).

[5] R. Y. Zhu, Proceeding of 4th Annual International Industrial Symposium on the Super Collider, J. Nonte, ed., Plenum Press , New York, 781 (1991).

[6] R. Novotny, Nucl. Phys. Proc. Suppl. 61B, 137 (1998)

[7] C.L. Woody, P.W. Levy and J.A. Kierstead, IEEE Transactions on Nuclear Science, 36, 536 (1989) 
154 [8] K. Sato et al., Poster presented at the 13th Vienna Conference on Instru-
mentation, http://indico.cern.ch/contributionDisplay.py?contribId=180.
155 [9] http://rmdinc.com/wp-content/uploads/2013/03/APD_Cut - Sheet

$157 \quad-$ MW_2 - 11 - 13.pdf

158 [10] M.Hoenk et al. Proc. SPIE, 915413 (2014).

159 [11] J. Hennessy et al, Applied Optics 54, 3507 (2015). 